\title{
Influence de l'enzyme coagulante de B. mesentericus souche 76 sur les fractions de la caséine par rapport aux autres enzymes d'origine microbienne ou animale
}

\author{
par \\ Liliana GORANOVA \\ et Maître de recherches Dr es Sciences biol. \\ Maria STEFANOVA-KONDRATENKO
}

Le manque de coagulant du lait d'origine animale (la présure) augmente d'année en année, ce qui accroît l'intérêt des produits de substitution. Des études en ce domaine sont entreprises dans plusieurs pays $[6,7,8,9,11,13,16,17,18,19,20,23,25,26]$.

Le problème du choix d'un remplaçant de la présure est très complexe, car chaque préparation qui sera employée dans ce but, doit être douée de qualités proches de celles de la présure elle-même. Un élément d'intérêt particulier est la manière dont agissent les substances en question sur la molécule de caséine et sur ces fractions. On en trouve diverses études dans ce domaine $[5,10,12,14,21]$.

\section{MATERIAUX ET METHODES DE TRAVAIL}

L'action de l'enzyme coagulant le lait, de Bac. mesentericus souche 76 , sur les fractions de la caséine a été étudiée au moyen de l'électrophorèse amidon-gel suivant la méthode d'Aschaffenburg $[1,2$, 3, 22, 29).

Pour comparaison, on a utilisé les substituants d'origine microbienne suivants :

MeIto obtenu de la moisissure Mucor pussilus Lindt, du Japon ;

RENNILASE préparation liquide de la moisissure Mucor miehei, du Danemark ;

Fromase préparation sèche obtenue du même producteur, mais en France ; 
Milcozyme préparation obtenue du Bac. polymixa, au Japon, et le coagulant liquide danois de la présure par l'entreprise "Lövengreen », Danemark, utilisé en tant que contrôle.

Pour l'étude de l'influence des produits cités, on utilise :

1) De la caséine acide, obtenue suivant la méthode de Seitov et Joumachev [27], sur laquelle on faisait agir séparément avec les si:préparations. Les quantités de solutions enzymatiques, employées pour l'étude de leur influence sur $2 \mathrm{ml}$ de solution de caséine à $5 \mathrm{p} .100$ varient de 0,05 à $0,1 \mathrm{ml}$. La durée d'action était de $15 \mathrm{mn}$ sous une température de $+35^{\circ} \mathrm{C}$. Les solutions de départ étaient de $0,5 \mathrm{p} .100$ pour les préparations sèches, de $5 \mathrm{p} .100$ pour la préparation de Rennilase et de 10 p. 100 pour le coagulant danois qui a servi de contrôle. Les préparations étaient faites de manière à assurer une activité adéquate pour toutes les préparations employées.

2) De la caséine acide, obtenue après traitement séparé des six préparations enzymatiques sur du lait de vache écrémé. Pour la coagulation du lait écrémé par les préparations on en a employé des quantités qui assuraient le départ de la coagulation de la $12^{\mathrm{e}}$ à la $15^{\circ} \mathrm{mn}$ et, uniquement pour E-76, de la $30^{\circ}$ à la $35^{\circ} \mathrm{mn}$.

De la caséine séchée on prenait $0,250 \mathrm{~g}$ que l'on faisait dissoudre dans $5 \mathrm{ml}$ de la solution tampon de tricitrate à $\mathrm{pH} 6,6$ et on ajoutait $2,5 \mathrm{~g}$ d'urée. On ajoutait à $1 \mathrm{ml}$ de solution une goutte de 2-mercaptoéthanol et, après $20 \mathrm{mn}$, on pratiquait l'essai. On retenait, pour contrôle, de la caséine non traitée par des préparations enzymatiques.

Avant d'obtenir de la caséine de la manière décrite, on laissait le coagulant en repos pendant $60 \mathrm{mn}$.

3) Du lait écrémé (pasteurisé ou non), traité séparément par les six préparations enzymatiques énumérées, suivant la méthode décrite, à l'exception de la E-76. Cette dernière était employée dans des quantités qui assuraient la coagulation après $10 \mathrm{mn}$, pour une partie des échantillons et de la $30^{\mathrm{e}}$ à la $35^{\mathrm{e}} \mathrm{mn}$ pour les autres.

$\mathrm{Du}$ coagulant, laissé à reposer pendant $60 \mathrm{mn}$ on prenait par $2 \mathrm{~g}$ et on ajoutait $1 \mathrm{~g}$ d'urée et 2 gouttes de 2 -mercaptoéthanol.

Avec une baguette de verre, on mélangeait jusqu'à pleine dissolution et on appliquait les échantillons sur des plaques pour électrophorèse après $20 \mathrm{mn}$. Pour contrôle, on se servait de lait écrémé, qui n'était pas traité par des préparations enzymatiques.

\section{RESULTATS}

Les électrophorégrammes 1 à 4 reflètent l'effet des préparations mentionnées sur les fractions de caséine acide, obtenue de la manière exposée dans le point 1 de la méthode. 


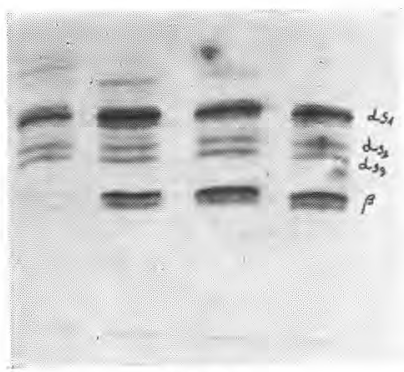

fig. 1

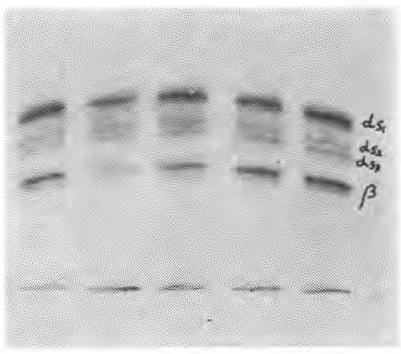

fig. 2

fig. 1

1) $2 \mathrm{ml}$ sol. 5 p. 100 cas. avec $0,05 \mathrm{ml} \mathrm{0,5}$ p. $100 \mathrm{E}-76,15 \mathrm{mn}\left(35^{\circ} \mathrm{C}\right)+2 \mathrm{ml} 8 \mathrm{M}$ urée.

2) $2 \mathrm{ml} \mathrm{sol.} 5$ p. 100 cas. avec $0,10 \mathrm{ml} 5 \mathrm{p} .100 \mathrm{P}, 15 \mathrm{mn}\left(35^{\circ} \mathrm{C}\right)+2 \mathrm{ml} 8 \mathrm{M}$ urée.

3) $2 \mathrm{ml}$ sol. 5 p. 100 cas, avec $0,10 \mathrm{ml} 10 \mathrm{p} .100 \mathrm{CD}, 15 \mathrm{mn}\left(35^{\circ} \mathrm{C}\right)+2 \mathrm{ml} 8 \mathrm{M}$ urée.

4) $2 \mathrm{ml}$ sol. 5 p. 100 cas. (non traité) $15 \mathrm{mn}\left(35^{\circ} \mathrm{C}\right)+2 \mathrm{ml} 8 \mathrm{M}$ urée.

fig. 2

1) $2 \mathrm{ml}$ sol. 5 p. 100 cas. avec $0,1 \mathrm{ml} 0,5$ p. $100 \mathrm{Mto}, 15 \mathrm{mn}\left(+35^{\circ} \mathrm{C}\right)+1,5 \mathrm{~g}$ urée $+2 \mathrm{ml} 8 \mathrm{M}$ urée.

2) $2 \mathrm{ml}$ sol. $5 \mathrm{p} .100 \mathrm{cas}$. avec $0,05 \mathrm{ml} \mathrm{0,5}$ p. $100 \mathrm{E}-76,15 \mathrm{mn}\left(35^{\circ} \mathrm{C}\right)+1,5 \mathrm{~g}$ urée $+2 \mathrm{ml} 8 \mathrm{M}$ urée.

3) $2 \mathrm{ml}$ sol. 5 p. 100 cas. avec $0,05 \mathrm{ml} \mathrm{0,5}$ p. $100 \mathrm{E}-76,15 \mathrm{mn}\left(35^{\circ} \mathrm{C}\right)+1,5 \mathrm{~g}$ urée $+2 \mathrm{ml} 8 \mathrm{M}$ urée.

4) $2 \mathrm{ml}$ sol. 5 p. 100 cas. avec $0,1 \mathrm{mil} 10$ p. $100 \mathrm{CD}, 15 \mathrm{mn}\left(35^{\circ} \mathrm{C}\right)+1,5 \mathrm{~g}$ urée $+2 \mathrm{ml} 8 \mathrm{M}$ urée.

5) $2 \mathrm{ml}$ sol. $5 \mathrm{p} .100 \mathrm{cas}$ (non traité) $15 \mathrm{mn}\left(35^{\circ} \mathrm{C}\right)+1,5 \mathrm{~g}$ urée $+2 \mathrm{ml} 8 \mathrm{M}$ urée.

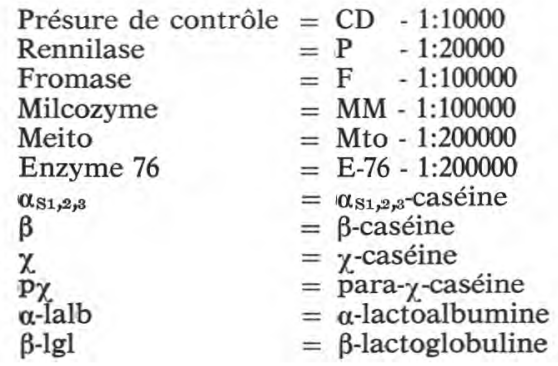

La figure 1 montre l'effet de la préparation E-76, de la Rennilase et de l'échantillon de contrôle avec coagulant contrôle sur les fractions de caséine, employées dans des quantités proportionnelles à leur activité coagulante, à l'exception de la E-76. Cette dernière est employée dans une quantité égale à 50 p. 100 de celle qui est calculée.

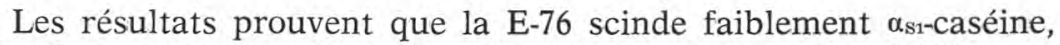
mais fortement $\beta$-caséine. La Rennilase scinde plus faiblement

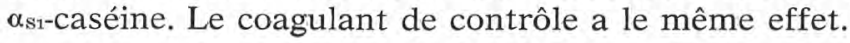




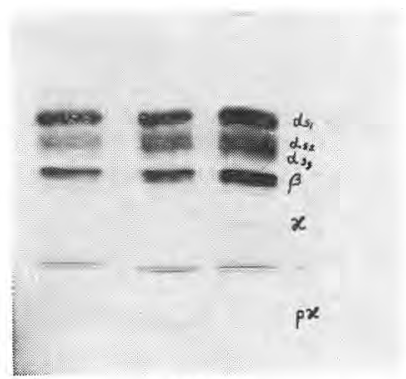

fig. 3

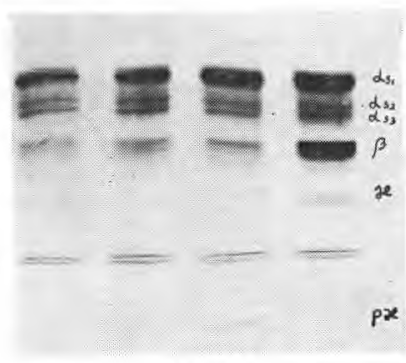

fig. 4

fig. 3

1) $2 \mathrm{ml} \mathrm{sol.} 5$ p. 100 cas. avec $0,1 \mathrm{ml} 1$ p. $100 \mathrm{~F} 15 \mathrm{mn}\left(35^{\circ} \mathrm{C}\right)+2 \mathrm{ml} 8 \mathrm{M}$ urée.

2) $2 \mathrm{ml} \mathrm{sol.} 5$ p. 100 cas. avec $0,1 \mathrm{ml} 10 \mathrm{p} .100 \mathrm{CD} 15 \mathrm{mn}\left(35^{\circ} \mathrm{C}\right)+2 \mathrm{ml} 8 \mathrm{M}$ urée.

3) $2 \mathrm{ml}$ sol. 5 p. 100 cas. (non traitée) $15 \mathrm{mn}\left(35^{\circ} \mathrm{C}\right)+2 \mathrm{ml} 8 \mathrm{M}$ urée.

fig. 4

1) $2 \mathrm{ml}$ sol. $5 \mathrm{p} .100$ cas. avec $0,05 \mathrm{ml} \mathrm{E}-76 / \mathrm{I} / 15 \mathrm{mn}\left(35^{\circ} \mathrm{C}\right), 10 \mathrm{mn}\left(75^{\circ} \mathrm{C}\right)$ $+2 \mathrm{ml} 8 \mathrm{M}$ urée.

2) $2 \mathrm{ml}$ sol. 5 p. 100 cas. avec $0,035 \mathrm{ml} \mathrm{E}-76 / \mathrm{II} / 15 \mathrm{mn}\left(35^{\circ} \mathrm{C}\right), 10 \mathrm{mn}\left(75^{\circ} \mathrm{C}\right)$ $+2 \mathrm{ml} 8 \mathrm{M}$ urée.

3) $2 \mathrm{ml}$ sol. $5 \mathrm{p} .100$ cas. avec $0,1 \mathrm{ml} \mathrm{MM} 15 \mathrm{mn}\left(35^{\circ} \mathrm{C}\right), 10 \mathrm{mn}\left(75^{\circ} \mathrm{C}\right)$ $+2 \mathrm{ml} 8 \mathrm{M}$ urée.

4) $2 \mathrm{ml}$ sol. $5 \mathrm{p} .100 \mathrm{cas}$ (non traitée) $15 \mathrm{mn}\left(35^{\circ} \mathrm{C}\right), 10 \mathrm{mn}\left(75^{\circ} \mathrm{C}\right.$ ) $+2 \mathrm{ml} 8 \mathrm{M}$ urée.

5) $2 \mathrm{ml}$ sol. 5 p. 100 cas. avec E-76/I/ - 1:200000 E-76/II/ - 1:300000 + $2 \mathrm{ml}$ $8 \mathrm{M}$ urée.

On peut remarquer que les produits de clivage, résultats de l'action de la Rennilase et du coagulant de contrôle, se rangent à une distance égale du point de départ, tandis que ceux de la E-76 à une distance un peu plus grande. Les lignes des produits scindés se trouvent au-dessus de $\alpha_{\text {si-caséine. }}$

La figure 2 montre l'effet de la E-76 sur la solution de 5 p. 100 de caséine pendant $15 \mathrm{mn}$ sous une température de $35^{\circ} \mathrm{C}$ et sur la même solution sans un repos de $15 \mathrm{mn}$, ainsi que l'effet des préparations Meito, et le coagulant de contrôle avec repos de $15 \mathrm{mn}$. On peut voir que la E-76 scinde plus la caséine dans les cas de repos de la solution $(15 \mathrm{mn})$. Le clivage de la caséine par la préparation Meito et par le contrôle est faible. La préparation Meito

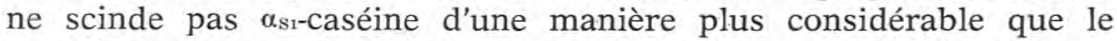
coagulant de contrôle. Les deux préparations ont un effet similaire sur $\beta$-caséine.

La figure 3 montre les modifications des fractions sous l'action des préparations Fromase et du coagulant de contrôle, employés dans une quantité proportionnelle à leur activité coagulante. La 


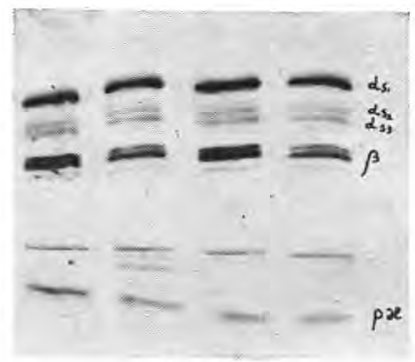

fig. 5

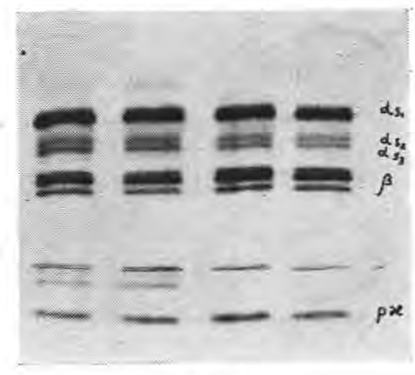

fig. 6

Echantillons de caséine isolée après traitement par des solutions enzymatiques sur du lait écrémé

fig. 5

1) Lait de vache avec Fromase

2) Lait de vache avec E-76

3) Lait de vache avec Contrôle

4) Lait de vache avec Rennilase coagul. pour $14 \mathrm{mn}\left(35^{\circ} \mathrm{C}\right)$ retient $60 \mathrm{mn}$. coagul. pour $22 \mathrm{mn}\left(35^{\circ} \mathrm{C}\right)$ retient $60 \mathrm{mn}$. coagul. pour $15 \mathrm{mn}\left(35^{\circ} \mathrm{C}\right)$ retient $60 \mathrm{mn}$. coagul. pour $12 \mathrm{mn}\left(35^{\circ} \mathrm{C}\right)$ retient $60 \mathrm{mn}$.

\section{fig. 6}

1) Lait de vache avec Milcozyme coagul. pour $15 \mathrm{mn}\left(35^{\circ} \mathrm{C}\right)$ retient $60 \mathrm{mn}$.

2) Lait de vache avec E-76

3) Lait de vache avec Contrôle coagul. pour $35 \mathrm{mn}\left(35^{\circ} \mathrm{C}\right)$ retient $60 \mathrm{mn}$. coagul. pour $15 \mathrm{mn}\left(35^{\circ} \mathrm{C}\right)$ retient $60 \mathrm{mn}$.

4) Lait de vache avec Meito coagul. pour $16 \mathrm{mn}\left(35^{\circ} \mathrm{C}\right)$ retient $60 \mathrm{mn}$.

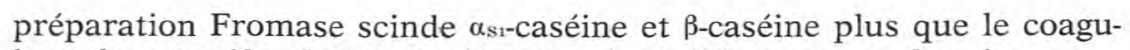
lant de contrôle. Cette dernière touche faiblement ces fractions.

L'électrophorégramme 4 montre l'effet parallèle des deux préparations, de la E-76 et de la préparation Milcozyme. Elles influencent

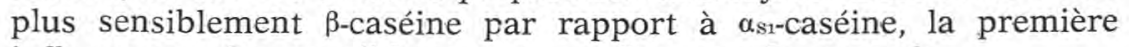
influençant plus sensiblement $\alpha_{\text {s1 }}$-caséine que la seconde.

Les électrophorégrammes 5 et 6 montrent l'image de la caséine, obtenue après traitement par les préparations E-76, Fromase, Rennilase, Milcozyme, Meito et le coagulant de contrôle du lait écrémé à température de $+35^{\circ} \mathrm{C}$. On constate une absence de différence dans l'effet de la préparation de Fromase et le coagulant de contrôle. Les préparations Rennilase, Milcozyme et E-76 scindent faiblement $\beta$-caséine, suivies par la préparation Meito. Les préparations E-76 et Milcozyme donnent deux lignes complémentaires de la caséine scindée, qui se manifestent immédiatement dès le départ.

Les figures 7 et 8 montrent l'effet des mêmes préparations sur le lait écrémé, sans séparation de la caséine. Les résultats prouvent un faible clivage de la caséine sous l'effet des préparations Milcozyme et E-76, plus sensiblement exprimé sous l'effet de la première. L'action 


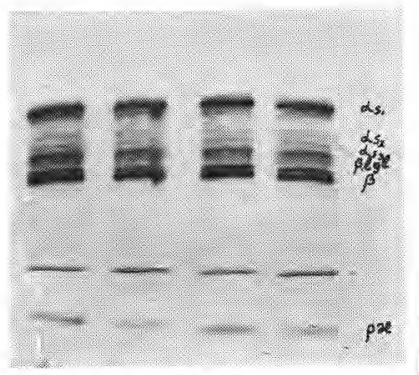

fig. 7

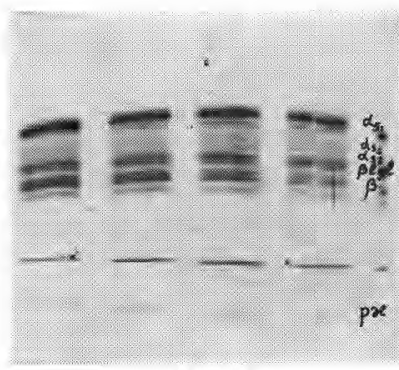

fig. 8

Echantillons de lait écrémé traité par des solutions enzymatiques sans séparation de la caséine qui en suit

fig. 7

1) Lait de vache avec Rennilase

2) Lait de vache avec E-76

coagul. pour $17 \mathrm{mn}\left(35^{\circ} \mathrm{C}\right)$ retient $60 \mathrm{mn}$. coagul, pour $32 \mathrm{mn}\left(35^{\circ} \mathrm{C}\right)$ retient $60 \mathrm{mn}$.

3) Lait de vache avec Contrôle coagul. pour $15 \mathrm{mn}\left(35^{\circ} \mathrm{C}\right)$ retient $60 \mathrm{mn}$.

4) Lait de vache avec Fromase coagul, pour $16 \mathrm{mn}\left(35^{\circ} \mathrm{C}\right)$ retient $60 \mathrm{mn}$.

fig. 8

1) Lait de vache avec Meito

2) Lait de vache avec Contrôle

3) Lait de vache avec E-76

coagul. pour $15 \mathrm{mn}\left(35^{\circ} \mathrm{C}\right)$ retient $90 \mathrm{mn}$. coagul. pour $15 \mathrm{mn}\left(35^{\circ} \mathrm{C}\right)$ retient $90 \mathrm{mn}$. coagul. pour $31 \mathrm{mn}\left(35^{\circ} \mathrm{C}\right)$ retient $90 \mathrm{mn}$.

4) Lait de vache avec Milcozyme coagul. pour $15 \mathrm{mn}\left(35^{\circ} \mathrm{C}\right)$ retient $90 \mathrm{mn}$.

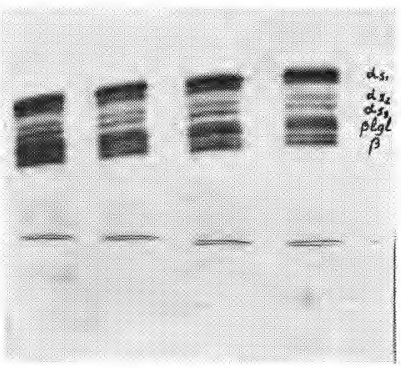

fig. 9

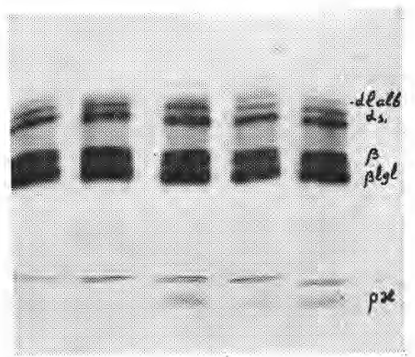

fig. 10

fig. 9

1) Lait de vache avec Rennilase coagul. pour $16 \mathrm{mn}\left(35^{\circ} \mathrm{C}\right)$ retient $60 \mathrm{mn}$.

2) Lait de vache avec Contrôle coagul. pour $15 \mathrm{mn}\left(35^{\circ} \mathrm{C}\right)$ retient $60 \mathrm{mn}$.

3) Lait de vache avec E-76 coagul. pour $35 \mathrm{mn}\left(35^{\circ} \mathrm{C}\right)$ retient $60 \mathrm{mn}$.

4) Lait de vache avec Fromase coagul. pour $15 \mathrm{mn}\left(35^{\circ} \mathrm{C}\right)$ retient $60 \mathrm{mn}$.

1) Lait de brebis cru.

fig. 10

2) Lait de brebis pasteurisé.

3) Lait de brebis pasteurisé avec Contrôle coagul. $16 \mathrm{mn}$ retient $60 \mathrm{mn}$.

4) Lait de brebis pasteurisé avec E-76 coagul. $35 \mathrm{mn}$ retient $60 \mathrm{mn}$.

5) Lait de brebis cru avec E-76 coagul. $34 \mathrm{mn}$ retient $60 \mathrm{mn}$. 


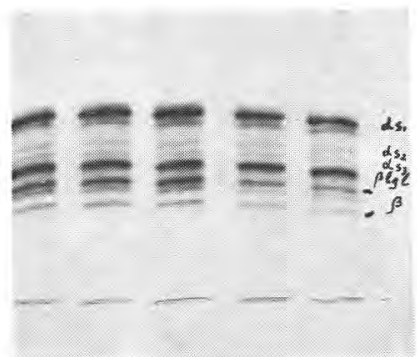

fig. 11

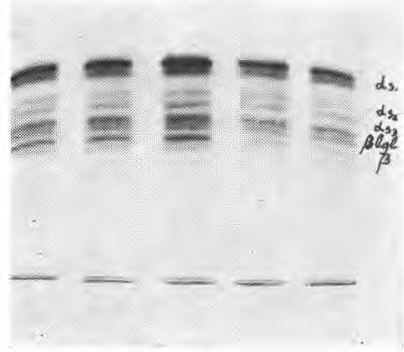

fig. 12

1) Lait de vache cru.

fig. 11

2) Lait de vache pasteurisé.

3) Lait de vache pasteurisé avec Contrôle coagul. $15 \mathrm{mn}$ retient $60 \mathrm{mn}$.

4) Lait de vache pasteurisé avec E-76 coagul. $28 \mathrm{mn}$ retient $60 \mathrm{mn}$.

5) Lait de vache cru avec E-76 coagul. $33 \mathrm{mn}$ retient $60 \mathrm{mn}$.

1) Lait de vache cru.

fig. 12

2) Lait de vache pasteurisé.

3) Lait de vache pasteurisé avec Contrôle coagul. $14 \mathrm{mn}\left(35^{\circ} \mathrm{C}\right)$ retient $60 \mathrm{mn}$.

4) Lait de vache pasteurisé avec E-76 coagul. $9 \mathrm{mn}\left(35^{\circ} \mathrm{C}\right)$ retient $60 \mathrm{mn}$.

5) Lait de vache cru avec E-76 coagul. $10 \mathrm{mn}\left(35^{\circ} \mathrm{C}\right)$ retient $60 \mathrm{mn}$.

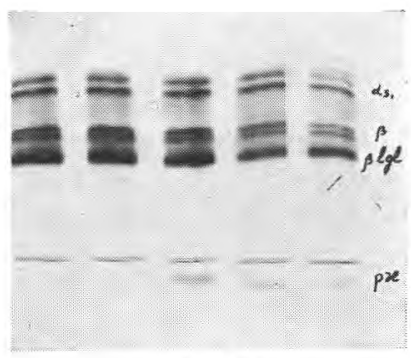

fig. 13

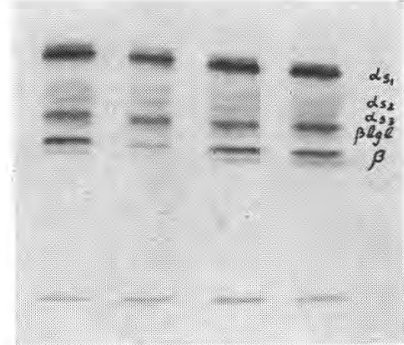

fig. 14

1) Lait de brebis cru.

fig. 13

2) Lait de brebis pasteurisé.

3) Lait de brebis pasteurisé avec Contrôle coagul. $15 \mathrm{mn}\left(35^{\circ} \mathrm{C}\right)$ retient $60 \mathrm{mn}$.

4) Lait de brebis pasteurisé avec E-76 coagul. $9 \mathrm{mn}\left(35^{\circ} \mathrm{C}\right)$ retient $60 \mathrm{mn}$.

5) Lait de brebis cru avec E-76 coagul. $8 \mathrm{mn}\left(35^{\circ} \mathrm{C}\right)$ retient $60 \mathrm{mn}$.

fig. 14

1) Lait de vache pasteurisé avec quant. normale de $\mathrm{CaCl}_{2}$ et Contrôle coagul. $15 \mathrm{mn}$.

2) Lait de vache pasteurisé avec quant. normale de $\mathrm{CaCl}_{2}$ et $\mathrm{E}-76$ coagul. $10 \mathrm{mn}$.

3) Lait de vache pasteurisé avec quant. double de $\mathrm{CaCl}_{2}$ et E-76 coagul. $10 \mathrm{mn}$.

4) Lait de vache pasteurisé avec quant. triple de $\mathrm{CaCl}_{2}$ et E-76 coagul. $10 \mathrm{mn}$.

Température de coagulation des quatre échantillons $35^{\circ} \mathrm{C}$ et $60 \mathrm{mn}$ de retenue. 
de E-76 provoque de nouveau l'apparition des bandes pâles de produits scindés sous la ligne de départ.

La figure 9 reflète l'effet des préparations Rennilase, Fromase, E-76 et le coagulant de contrôle sur le lait de vache traité par ces préparations et retenu pendant $60 \mathrm{mn}$. Uniquement la préparation Fromase mène à une faible modification de $\alpha_{\mathrm{S}_{1} \text {-caséine. }}$

Les électrophorégrammes $10,11,12$ et 13 reflètent l'effet de E-76 sur du lait cru et du lait pasteurisé de vache et de brebis, en employant des quantités qui assurent le déroulement de la coagulation entre la $30^{\circ}$ et la $35^{\circ} \mathrm{mn}$, et à la $10^{\circ} \mathrm{mn}$ avec retenue de la coagulation pendant $60 \mathrm{mn}$. Les résultats montrent que, lors d'une coagulation entre la $30^{\circ}$ et la $35^{\mathrm{e}} \mathrm{mn}$ du lait pasteurisé de brebis, du lait cru

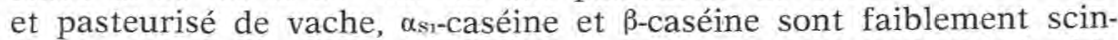
dées. Ceci est mieux exprimé dans le lait cru et pasteurisé de brebis et de vache quand la coagulation a lieu à la $10^{\mathrm{e}} \mathrm{mn}$. Le lait cru de brebis ne manifeste pas des modifications de ses fractions sous l'influence de la E-76 quand la coagulation a lieu entre la $30^{\mathrm{e}}$ et la $35^{\mathrm{e}} \mathrm{mn}$.

Dans le dernier électrophorégramme (fig. 14) est montré l'influence de la quantité normale, ainsi que de la quantité doublée et triplée de bichlorure de calcium sur l'effet de E-76. L'image prouve que la coagulation par E-76 avec addition d'une quantité normale de bichlorure de calcium, en respectant toutes les conditions mentionnées, est due à la présence d'un plus considérable clivage de

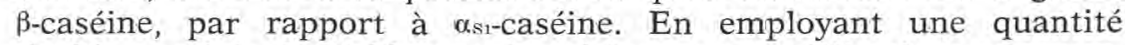
double et triple de bichlorure de calcium, ces modifications apparemment disparaissent.

\section{CONCLUSIONS}

1) Sous l'influence de E-76, dans des concentrations proportionnelles à son activité coagulante, on est témoin d'un clivage dans la caséine des fractions de $\alpha_{\mathrm{si}}$ et de $\beta$-caséine. De la même manière agit aussi la préparation Milcozyme. Les deux préparations, au cours du clivage de la caséine, donnent deux lignes complémentaires de produits scindés, qui se manifestent immédiatement dès le départ.

2) L'image électrophorétique de la caséine, obtenue après l'action des préparations Rennilase, Fromase, Milcozyme, E-76, Meito et le coagulant de contrôle, montre un plus haut degré de clivage de $\beta$-caséine par les préparations E-76, Milcozyme, Rennilase, par rapport

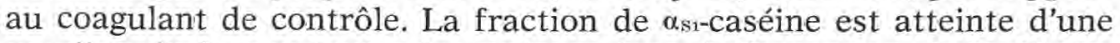
manière égale par toutes les préparations, à l'exception de la Meito. Cette dernière a une faible influence sur cette fraction.

3) L'image électrophorétique du fromage de lait de vache, obtenu sous l'effet des préparations étudiées, montre que E-76 et la prépa- 
ration Milcozyme scindent faiblement $\beta$-caséine. La fraction de

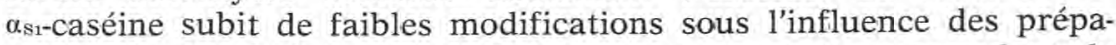
rations Meito, Milcozyme et Fromase, par rapport au coagulant de contrôle et à la préparation Rennilase.

4) Quand la coagulation du lait de vache (cru et pasteurisé) avec E-76 est menée jusqu'à la $35^{\circ} \mathrm{mn}$ et que le fromage est retenu $60 \mathrm{mn}$, on est témoin des faibles modifications des fractions de $\alpha_{\text {s1-caséine }}$ et $\beta$-caséine, par rapport à l'action du coagulant de contrôle. Dans les cas où la coagulation a lieu jusqu'à la $10^{\mathrm{e}} \mathrm{mn}$ et où le fromage est retenu pendant $60 \mathrm{mn}$, on parvient au clivage de toutes les fractions de la caséine du lait de vache cru et pasteurisé. La caséine du lait de brebis est scindée faiblement dans les mêmes conditions (les modifications de $\beta$-caséine est plus considérable par rapport au coagulant de contrôle).

5) Le lait de vache cru et pasteurisé, traité par E-76, montre un cli-

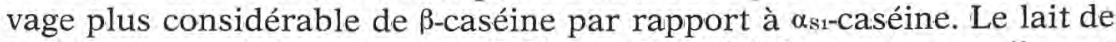
brebis pasteurisé, traité par E-76, modifie faiblement $\alpha_{\mathbb{S} 1 \text {-caséine et }}$ $\beta$-caséine par rapport au coagulant de contrôle. Dans le lait de brebis cru, ces modifications n'ont pas lieu.

6) L'électrophorégramme du fromage de lait de vache, dans lequel on a ajouté une quantité double ou triple de chlorure de calcium et où la coagulation est menée jusqu'à la $10^{\mathrm{e}} \mathrm{mn}$, le clivage

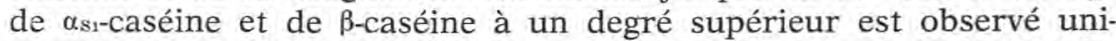
quement dans l'échantillon avec la concentration normale du bichlorure de calcium. En augmentant les concentrations du bichlorure de calcium, les modifications dans la caséine du lait de vache, sous l'action de E-76 et du coagulant de contrôle, manquent.

\section{S u m m a r y}

\section{INFLUENCE OF THE COAGULATING ENZYME OF STRAIN B. MESENTERICUS 76 ON THE CASEIN FRACTIONS COMPARED TO THAT OF OTHER ENZYMES OF MICROBIAL OR ANIMAL ORIGIN}

The influence of the bacterial rennet substitutes E-76 and Milkosim as well as that of the enzyme preparations of mould origin Renelase, Fromase and Meito on the $\alpha_{\mathbf{s}_{1}}$ and $\beta$-casein fractions was investigated. A liquid animal rennet was used as a control. The investigation was carried out applying starch-gel-electrophoresis.

Casein solutions at $\mathrm{pH} \mathrm{6,6} \mathrm{were} \mathrm{treated} \mathrm{with} \mathrm{the} \mathrm{above} \mathrm{mentioned}$ substitutes in concentrations proportional to the established coagulating activities. Degradation of $\alpha_{\mathrm{s}^{-}}$and $\beta$-casein fractions was stronger when these were treated with E-76 and Milkosim.

The use of E-76 and the control in the quantities commonly applied in cheese making resulted in a similar degradation pattern. 
The degradation of the $\beta$-casein fraction by Milkosim was very similar to that caused by E-76.

The changes which the $\alpha_{\text {s1 }}$ fraction of casein obtained after treatment of skim milk with Renelase, Fromase, Milkosim and E-76 undergo were equivalent irrespective of the reagent used.

The investigation of the curd obtained using the above substitutes gave the following results:

a) insignificant decrease of $\beta$-casein fraction when using the preparations Milkosim and E-76 compared to that resulting by using the control;

b) identical changes of $\alpha_{\text {s1 }^{-}}$and $\beta$-casein fractions treated with Renelase, Fromase, Meito and the control successively.

The influence of E-76 and the control on both pasteurized and raw sheep and cow-milk as well as that on milk with increalsed calcium chloride content was investigated.

Reçu pour publication le 14 juin 1974. 